\title{
Government public health intelligence units: bridging the data to policy gap
}

\section{Cynthia R Driver ${ }^{1}$, Ruxana Jina ${ }^{1}$, Cristian A Herrera ${ }^{2,3}$, Carla Abouzhar ${ }^{4}$, Lorna E Thorpe ${ }^{5}$, Adam Karpati ${ }^{1}$}

\footnotetext{
${ }^{1}$ Vital Strategies, New York City, New York, USA

${ }^{2}$ Health Division, Organisation for Economic Cooperation and Development, OECD, Geneva, Switzerland

${ }^{3}$ Department of Public Health, School of Medicine, Pontificia Universidad Católica de Chile, Santiago, Chile

${ }^{4}$ CAZ Consulting, Geneva, Switzerland

${ }^{5}$ NYU School of Medicine Department of Population Health, New York City, New York, USA
}

Research has shown that the use of public health data for policymaking and planning has remained low in low and middle-income countries. Public health intelligence services can bridge this gap between data analysis and policy decision-making.
$\mathrm{P}$ ublic health policymaking and planning are core government functions. Sound health policy requires rigorous and objective analysis of data that identifies needs, weighs alternative strategies, and generates usable information to guide policy decisions. Literature from low and middle-income countries has shown that despite technological advances, the actual use of data remains low [1]. Barriers include: 1) limited resources to analyse data; 2) reluctance to use data to highlight problems or support decisions that contradict priorities of interest group or political preferences; 3) data are not always available to address problems that policymakers are facing; 4) limited communication between data producers and data users; 5) data are not always easy for policymakers to understand and use; and 6) data are not always valued, due to concerns about quality and validity [1]. As a result, analyses that are conducted often do not gain policy traction.

Public health intelligence (PHI) services provide an opportunity to bridge the gap between data analysis and policy decision-making. We propose that PHI services should be strengthened within government health institutions. Furthermore, we believe that the most efficient, effective, and sustainable way to deliver these services is through a dedicated organizational unit that integrates potentially fragmented efforts [2].

\section{WHAT ARE PHI UNITS?}

The definition of 'PHI' has evolved as the discipline has become more established. It commonly includes the development of knowledge through compiling and analysing data from multiple sources so that it can be used to take action [3]. PHI provides information on determinants and patterns of health and disease and on the functioning of the health care system. The activities of PHI units include monitoring and producing reports on population-based health status, health situation analysis, health impact assessment, surveys, health equity analysis, and economic analysis. They also set standards for data analysis and dissemination and build institutional capacity. Depending on capacity, PHI units could also include health technology assessments and clinical practice guidelines. 


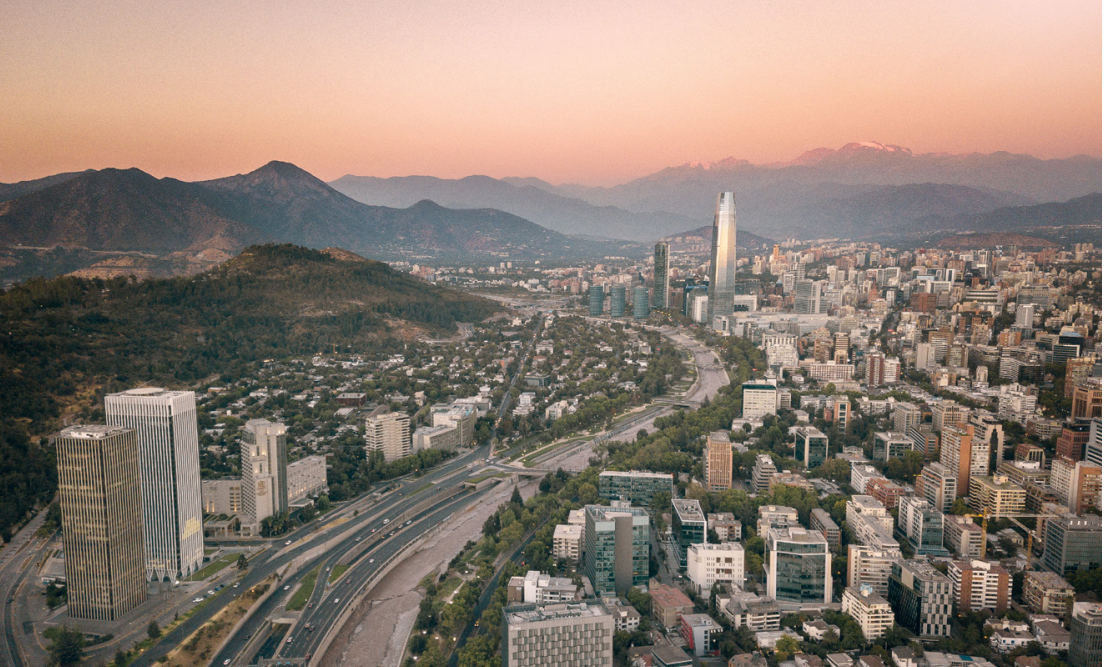

Photo: The Division of Health Planning in the Chile Ministry of Health functions as a public health intelligence unit (purchased from Shutterstock).
PHI services are delivered in a variety of structures and settings. For example, the term health observatory has sometimes been used to describe surveillance hubs, whereas it has also been used to describe units in which analysts focus on integrating and analysing data for decision-making, hosted in either government or non-government settings. Observatories tend to be comparatively autonomous and typically have strong networks for accessing data. In practice though, the focus of observatories is often topic- or subpopulation-specific and may represent a narrower application of the PHI concept [4].

Other locations of health surveillance include public health institutes and epide-

miology units. In many settings, policy and planning units, monitoring and evaluation units and research institutes also perform PHI functions. In this paper, we use PHI unit as a more general term to refer to specialized organizational structures that bring together analytic expertise and multiple data sources to provide policy-relevant analyses for immediate use and public health action.

Globally, there are examples of PHI services in government or other settings. However, we believe data to policy efforts are at greater risk of not being sustained when they are not housed in government. Below, we present some of the reasons for implementing the model of embedding PHI units within government.

\section{GOVERNMENT AS AN INSTITUTIONAL SETTING FOR PHI FUNCTIONS}

The role of government in establishing PHI units for policy making is not always considered [3]. Although there are several key arguments against government hosting of PHI functions, there are a number of compelling reasons that may outweigh these concerns. Key concerns include the lack of expertise and human resource capacity, the investment needed to establish units, the constraints of achieving change within large government institutions, and finally, the potential susceptibility of government agencies to political influence.

Yet, there are several views that support the establishment of PHI units in government. Most importantly, government ownership of PHI increases public accountability. Although formal separation of statistical functions from policy making is recommended for ensuring scientific independence, the placement of PHI functions in a government setting has the potential to hold governments more accountable to national priorities. When PHI is more proximate, policy-making can be more attuned to current realities and real-time shifts in priorities. Maintaining accountability while enabling independence from political influence could be achieved through regulatory or procedural approaches. For example, principal statistical agencies in the United States have the authority to release statistical information without prior clearance [5].

Second, developing such units provides a capacity-building opportunity within government. PHI units build skills in data collection, analysis and dissemination in countries where such expertise is strongly needed. Such investment creates expertise, whereas diversion of resources away from public institutions simply reinforces the lack of expertise [6]. While academic partnerships can provide efficiency, the costs for such services can be prohibitive.

A third reason is to improve data quality. Government administrative systems are major data sources. The greater analysis and use of data locally by governments also lead to improved data quality. Country-specific examples suggest that prompt recognition of the sources of data quality problems is possible with regional analysis of mortality data [7]. Greater institutional proximity between data collection, compilation, validation, analysis and use functions promote better data quality. 
Fourth, integrating PHI functions into government agencies provides greater opportunity to integrate data analysis with policy making. Policymakers benefit from having immediate access to staff who can generate reliable information with maximum knowledge of operational and political contexts. Merging PHI functions within a single unit is likely to have a greater impact than dispersed efforts.

Fifth, when the data compilation and integration function lie within a government public health agency, access and collaboration with other government entities are facilitated. These collaborations are critical for contemporary public health practice. Indeed, when policymakers are actively engaged in the development of data products, they tend to trust the results more. Furthermore, this can also facilitate government collaboration with non-governmental actors, creating synergies that can benefit both sides and improve knowledge generation.

\section{CASE STUDIES}

\section{Chile Ministry of Health, Division of Health Planning}

Within the Public Health Secretariat of the Chile Ministry of Health, most PHI functions are the responsibility of the Division of Health Planning. Its mission is to manage health related data in order to generate knowledge to inform public policymaking at the national and regional levels [8]. The division's responsibility includes health statistics, health economics, national health strategy, health technology assessment and evidence-based health care, and epidemiology. As an example, in 2017, the Unit provided results on the effectiveness, economic evaluations, and social and ethical considerations for 56 treatments and diagnostic tests. The Ministers of Health and Finance used this information to prioritize and select treatments that should be covered by the financial protection system. As a result, a number of new high-cost medicines, medical devices and diagnostic tests were included [9].

\section{Ministry of Health of Morocco, Regional Health Observatories}

As part of a process to decentralize administrative services of government, which began in 2015, observatories were established in the twelve regions of Morocco to support the effective use of data and public health planning by the regional health directors [10]. The mandate of the regional health observatories includes the collection, analysis and assessment of health information, monitoring of key indicators, development of reports on health status and health services, participation in health studies, management of health information systems and participation in capacity building of health professionals. The observatory staff have competencies in epidemiology, public health, statistics, and data management and informatics. Because of their mandate and skills of their staffs, the observatories are able to collaborate with key partners within, and outside, of government to fully utilize health data. Already, staff have identified gaps in data regarding under reporting from the private sector and are developing strategies to correct this.

\section{CONCLUSION}

PHI is critically important for public health policy and planning aimed at promoting and protecting the health of its citizens. Although the range of skills and organizational placement can vary, we argue that policy-relevant PHI functions should be housed in government structures that have a strong relationship with and role in policymaking. There are a number of factors that would need to be considered when setting up a government PHI unit. Establishing the unit will require leadership, commitment and adequate finances for the sustainable functioning. The formation of such a unit will also be more successful within a dynamic public health sector where there

Public health intelligence services need to be strengthened in government and the most efficient, effective and sustainable way of achieving this is through a dedicated organizational unit. is a willingness to adapt the organizational structure.

The development of PHI within government does not require a significant initial investment; rather it can be achieved incrementally over time. Initially, more commonly used basic analyses important for day-to-day decision-making and that provide public policy information can be developed, while the more specialized analysis functions may be conducted in partnership with non-governmental organizations such as institutes or universities where methodologic expertise is available. The more specialized skills can then be procured or strengthened over time. 
Effective policy is developed from accurate and relevant data. PHI units are an effective and sustainable mechanism to ensure that governments have the tools and information required to develop policy that is responsive to national health priorities.

Disclaimer: The opinions expressed in the paper are the responsibility of the authors and do not necessarily reflect those of the OECD or its member countries.

Funding: This work was supported by the Bloomberg Philanthropies Data for Health Initiative.

Authorship contributions: CRD, RJ and CAH were responsible for drafting the manuscript. CA, LET and AK provided and conceptual guidance and reviewed the paper.

Competing interests: The authors completed the ICMJE Unified Competing Interest form (available upon request from the corresponding author), and declare no conflicts of interest

1 Li M, Brodsky I, Geers E. Barriers to Use of Health Data in Low- and Middle- Income Countries-A Review of the Literature. North Carolina: MEASURE Evaluation; 2018.

2 Gattini CH. Implementing National Health Observatories: operational approach and strategic recommendations. Santiago de Chile: Pan American Health Organization, Regional Office of the World Health Organization; 2009.

3 Jacobson B, Castillo-Salgado C. Providing health intelligence to meet local needs: a practical guide to serving local and urban communities through public health observatories. Kobe: World Health Organization; 2014.

4 World Health Organization. Urban health observatories: A possible solution to filling a gap in public health intelligence. Kobe: World Health Organization; 2013.

5 Principal Statistical Agencies. Statement of Commitment to Scientific Integrity. Washington: Federal Committee on Statistical Methodology; Undated.

6 Boerma T, Victora C, Abouzahr C. Monitoring country progress and achievements by making global predictions: is the tail wagging the dog? Lancet. 2018;392:607-9. Medline:29661480 doi:10.1016/S0140-6736(18)30586-5

7 Bloomberg MR, Bishop J. Understanding death, extending life. Lancet. 2015;386:e18-9. Medline:26434355 doi:10.1016/ S0140-6736(15)00400-6

8 Ministerio de Salud. Organization chart. Ministerio de Salud - Gobierno de Chile. Available: http://www.minsal.cl/organigrama/. Accessed: 5 September 2018.

9 Ministerio del Interior y Seguridad Pública. Determina Los Diagnósticos y Tratamientos De Alto Costo Con Sistema De Protección Financiera De La Ley No 20.8500. Dec 29, 2017.

10 Resolution of the Minister of Health regarding the creation and specification of the terms of reference and organization of decentralized interests of the Ministry of Health No. 16-003 of 23 Rabi' al-Awwal 1437 (4 January 2016).

\section{Correspondence to:}

Ruxana Jina, MBChB, MMed, PhD

Vital Strategies

100 Broadway, 4th Floor

New York, NY 10006 USA

rjina@vitalstrategies.org 